\title{
High-quality reports and their characteristics in the Japanese Adverse Drug Event Report database (JADER)
}

\author{
Masami Tsuchiya ${ }^{1,2}$, Taku Obara ${ }^{3,4}$, Makoto Miyazaki ${ }^{2}$, Aoi Noda ${ }^{3,4}$, Takamasa Sakai ${ }^{5}$, Ryohkan Funakoshi ${ }^{6}$, and \\ Nariyasu Mano ${ }^{2,3}$ \\ ${ }^{1}$ Department of Pharmacy, Miyagi Cancer Center and ${ }^{2}$ Laboratory of Clinical Pharmacy, Tohoku University Graduate \\ School of Pharmaceutical Sciences; ${ }^{3}$ Department of Pharmaceutical Sciences, Tohoku University Hospital; ${ }^{4}$ Division of \\ Preventive Medicine and Epidemiology, Tohoku University Tohoku Medical Megabank Organization; ${ }^{5}$ Drug \\ Informatics, Faculty of Pharmacy, Meijo University; ${ }^{6}$ Department of Pharmacy, Kameda General Hospital
}

Corresponding Author: Taku Obara, Ph.D., Division of Preventive Medicine and Epidemiology, Tohoku Medical Megabank Organization, 2-1, Seiryo-machi, Aoba-ku, Sendai, Miyagi 980-8573, Japan; TEL: (+81)-22-717-8104; Fax: (+81)-22-717-8106; email: obara-t@hosp.tohoku.ac.jp.

Received, March 3, 2021; Revised March 24, 2021; Accepted, March 24, 2021; Published, April 8, 2021

ABSTRACT -- Purpose: Spontaneous adverse drug reaction reporting is the foundation of postmarketing
drug safety monitoring. The present study aimed to analyze and clarify the quality and characteristics of the
Japanese Adverse Drug Event Report database (JADER) using the World Health Organization (WHO)
documentation grading scheme and the vigiGrade completeness score. The characteristics of reports were
described using both schemes simultaneously. The way of proper use of these two schemes was explored.
Methods: The WHO documentation grading scheme and the vigiGrade completeness score were applied to
the same dataset (JADER202001 dataset). Reports classified as high-quality under both assessment criteria
were extracted, and the characteristics of these reports were analyzed. Results: Of the 607,361 adverse drug
reaction reports analyzed, $52.8 \%$ were 'well-documented reports' with a vigiGrade completeness score $>0.8$.
Under the WHO documentation grading scheme, 328,702 reports (54.1\%) were Grade 2 and $5,178(0.9 \%)$
were Grade 3 (including rechallenge information). Among well-documented Grade 3 reports, classified as the
highest quality, a high proportion of the adverse drug reaction reports were related to disorders of
hematopoietic function resulting from anticancer drugs. Because a high proportion of the reports with
rechallenge information were for anticancer drugs as suspect drugs, the WHO documentation grading scheme
tended to extract reports regarding anticancer drugs as high quality. Conclusions: We conclude that the two
schemes need to be used appropriately, depending on the purpose of analysis, the target adverse drug reactions,
and suspect drugs.

\section{INTRODUCTION}

The collection, evaluation, and use of drug safety information by healthcare professionals are essential for the safe and proper use of drugs in clinical settings. Healthcare professionals, pharmaceutical companies, and regulatory authorities are required to carry out pharmacovigilance activities as part of a consistent set of safety measures from the drug development to postmarketing stages. Spontaneous reporting of adverse drug reactions (ADR) is the foundation of postmarketing drug safety monitoring $(1,2)$. It can be defined as a system for collecting and collating case reports of suspected ADRs to detect the potential for unknown drug-related harmful effects. Some of the ADR reports collected in Japan are made public via the Japanese Adverse Drug Event Report database (JADER database). They can be downloaded from the Pharmaceuticals and Medical Devices Agency (PMDA) website (3).
Similar databases are maintained in other countries and organizations around the world, including: the VigiBase, maintained by the WHO (4); FAERS (FDA Adverse Event Reporting System), maintained by the United States Food and Drug Administration (FDA) (5); the Lareb database, maintained by the Netherlands Pharmacovigilance Centre Lareb (6); and Eudravigilance, maintained by the EMA (European Medicines Agency) (7). Through signal detection and other tools, these databases play an essential role in postmarketing drug safety.

The quality of ADR information used for causality assessment and signal detection can be assessed by a pharmacoepidemiology expert or by evaluating the data elements' completeness in each case report. Completeness assessment tools include: the WHO's documentation grading scheme (8); vigiGrade (9), which was created to assess the quality of the information in VigiBase; the ATHE 
score (10); amongst other methods of calculating completeness scores (11-13). Here, we have analyzed the quality and characteristics of the JADER, using the WHO documentation grading scheme (14) and the vigiGrade (15). The WHO's documentation grading scheme, which was noted if essential elements (e.g., date of onset of ADR, duration of administration of suspected drugs, dose, and frequency of administration, rechallenge information) were given in the reports, could divide ADR reports into four grades. The vigiGrade completeness score was developed to measure the amount of clinically relevant information in a structured format without reflecting whether the information establishes causality between the drug and ADR (15). In this study, we compared and contrasted the characteristics of reports using the two schemes. Besides, the proper use of these two schemes was explored. This study aimed to find a way to evaluate the quality of ADR reports for postmarketing safety measures effectively.

\section{METHODS}

For this study, the WHO documentation grading scheme (8) and the vigiGrade completeness score (9) were applied to the same dataset (JADER202001). Reports classified as high-quality under both assessment criteria were extracted, and the characteristics of these reports were analyzed. The JADER202001 dataset containing ADR reports from April 2004 to September 2019 was used. JADER uses a report format based on ICH E2B-M2 (R3) and consists of four tables: 1) case list table (demo) (611,336 entries); 2) drug information table (drug) (3,571,439 entries); 3) ADR information table (reac) (966,444 entries); and 4) underlying disease table (hist) (1,231,770 entries). From the JADER202001 dataset, the data for the first listed suspect drug (in 607,361 reports) and the data for the first listed ADR (in 611,336 reports) were extracted and connected. All 607,361 connected cases were subsequently analyzed in a similar manner. All ADRs and underlying diseases were coded using the Medical Dictionary for Regulatory Activities (MedDRA) terminology (Version 22.1) preferred term (PT).

The WHO documentation grading scheme (8) classifies ADR reports using a 4-grade scheme, allowing classification from Grade 0 (lowest quality) to Grade 3 (highest quality); the algorithm for this scheme is shown in Figure 1. A report is classified as Grade 3 if it includes information on rechallenge (re- administration of the suspect drug after the onset of the ADR), the suspect drug start date, ADR onset date, the reason for the use of the suspect drug, and outcome of ADR. In the vigiGrade completeness score (9), a score of the completeness of a fixed number of elements included in the ADR report, can be calculated using the formula shown below:

Completeness score

$$
=\prod_{i=1}^{10}\left(1-P_{i}\right)=\left(1-P_{1}\right) \ldots\left(1-P_{10}\right)
$$

Where $P_{\mathrm{i}}$ is a numerical penalty for the elements $i$ shown in Table $\mathrm{S} 1$.

The elements included in the JADER were used for calculating the vigiGrade completeness score. Time-to-onset was calculated from the suspect drug start date and the ADR onset date and used in the vigiGrade completeness score calculation (Figure S1). As with our previous research (15), some of the assessed elements and penalties used in this study were modified from the original vigiGrade completeness score (Table S1). In previous research by the WHO Uppsala Monitoring Centre (9), a vigiGrade completeness score of $>0.8$ was defined as a 'well-documented report'. The same definition was used in this study. Significance was defined as $\mathrm{p}<0.05$ for all statistical tests, and SAS version 9.4 (SAS Institute Inc., Cary, NC, USA) was used for all analyses.

\section{RESULTS}

Of the 607,361 ADR reports analyzed, 320,657 $(52.8 \%)$ were 'well-documented reports' with a vigiGrade completeness score $>0.8$ (9). Under the WHO documentation grading scheme (8), 328,702 (54.1\%) were Grade 2 and 5,178 (0.9\%) were Grade 3. Table 1 shows the relationship between the vigiGrade completeness score and the WHO documentation grading. According to the results, 315,616 reports $(52.0 \%)$ were classified as welldocumented and Grade 2, and 5,041 reports (0.8\%) were well-documented and Grade 3. Moreover, all 'well-documented reports' according to vigiGrade were classified as Grade $\geq 2$ under the WHO documentation grading scheme. There were 233,569 not well-documented Grade 0 reports, representing $38.5 \%$ of the overall reports.

The report characteristics based on the combined vigiGrade completeness score and the WHO documentation grading scheme are presented in Table 2. In addition, the percentages of missing data are presented in Table 3. Among reports classified as 
both well-documented and Grade 3, a physician was included among the reporters in 4,260 reports $(84.5 \%)$, and 4,889 reports $(97.0 \%)$ came from a pharmaceutical company. The report status was 'investigation complete' in 5,040 reports (99.9\%), and spontaneous reports accounted for 3,468 (68.8\%) of all well-documented Grade 3 reports. Analysis of missing elements showed that there were almost no omissions in well-documented Grade 3 reports. In contrast, among the not well-documented Grade 0 reports, none allowed time-to-onset to be calculated, and all were unclear about whether the suspect drug was used before or after the ADR occurred. The not well-documented Grade 1-3 reports might omit more than one essential (penalty $50 \%$ ) or important (30\% penalty, if missing) element, such as time-to-onset, sex, age, etc.

In the overall reports, the top ten primary suspect drugs included immunosuppressants such as methotrexate, tacrolimus, prednisolone, and cyclosporine, and anticoagulants rivaroxaban and apixaban (Table S2). However, among welldocumented Grade 3 reports, eight of the top ten primary suspect drugs were anticancer drugs, and the other two were nafamostat mesylate and irradiated platelet concentrate. ADRs common in both classifications were cytopenias (such as neutropenia and thrombocytopenia), anaphylactic shock, hepatic impairment, and drug eruption. Although interstitial lung disease and pneumonia were two of the top ten in the overall reports, these ADRs were not in the top ten in well-documented Grade 3 reports. Pyrexia, diarrhea, and decreased blood pressure were not in the top ten in the overall reports (Table S3).

Among well-documented Grade 3 reports, the most common pair of the suspect drug and ADR was paclitaxel and leukopenia $(n=61,1.2 \%$; Table S4). Among not well-documented and Grade 0 reports, classified the lowest quality reports, immunosuppressive drugs, anticoagulants, and an antiepileptic drug (carbamazepine) were in the top ten suspect drugs similar to the overall trend. The top ten adverse drug reactions included interstitial lung disease, pneumonia, renal dysfunction, lymphoproliferative disorders, and death.Methotrexate and lymphoproliferative disorders were the most common pair of the suspect drug and ADR among not well-documented and Grade 0 reports ( $n=2,036,0.9 \%$; Table $S 4)$.

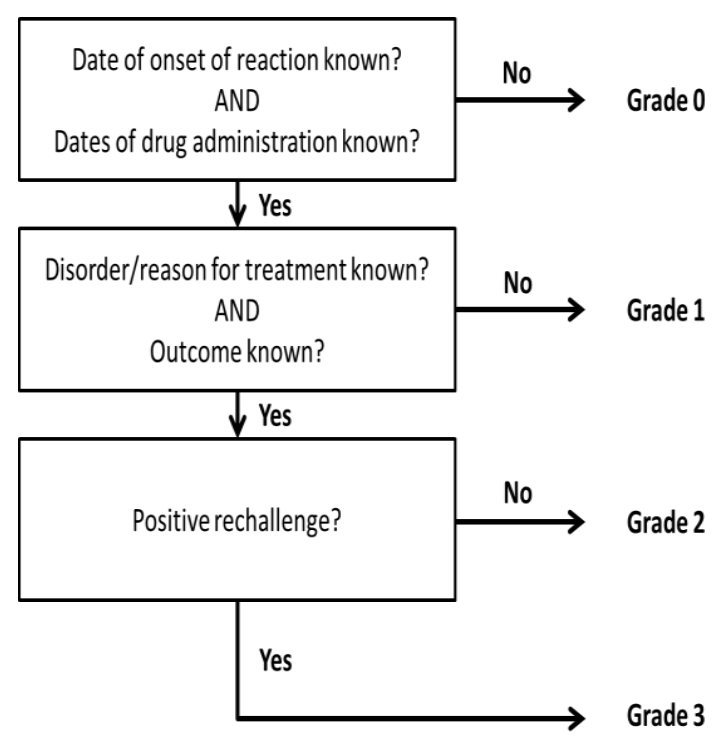

Figure 1. The documentation grading scheme developed by the World Health Organization

\section{DISCUSSION}

\section{High-quality ADR reports across the two assessment criteria}

In the present study, the WHO documentation grading scheme (8) and the vigiGrade completeness score (9) were used to extract high-quality reports in common across the two assessment criteria and explore their characteristics. All of the 'welldocumented reports' in the vigiGrade were classified as Grade 2 or above in the WHO documentation grading scheme. The WHO documentation grading scheme only evaluated the presence or absence of information, but not the information's granularity or accuracy (as evaluated by vigiGrade). Welldocumented reports judged by the vigiGrade could have enough information such as outcome or indication, which were essential to be classified into grade 3 or 4 by the WHO's grading scheme (8). 
Table 1. Relationship between vigiGrade 'well-documented reports' and WHO documentation grading $(n=607,361)$

\begin{tabular}{ccc}
\hline & \multicolumn{2}{c}{ vigiGrade } \\
\hline WHO documentation grading & Well-documented & Not well-documented \\
\hline Grade $0, \mathrm{n}(\%)$ & $0(0)$ & $233,569(38.5)$ \\
Grade 1, $\mathrm{n}(\%)$ & $0(0)$ & $39,912(6.5)$ \\
Grade 2, $\mathrm{n}(\%)$ & $315,616(52.0)$ & $13,086(2.1)$ \\
Grade 3, $\mathrm{n}(\%)$ & $5,041(0.8)$ & $137(0.1)$ \\
\hline
\end{tabular}

This was a potential reason explaining why a 'well-documented report' was considered high quality across the two evaluation criteria. Whereas, because the vigiGrade completeness score focused on time-to-onset for quality evaluation, some ADR reports with fewer missing elements were classified as not well-documented due to omitting time-toonset information.

\section{The characteristics of 'well-documented' Grade 3 reports}

Well-documented Grade 3 reports were analyzed to extract the highest-quality reports. Under the WHO documentation grading scheme (8), Grade 3 requires information on rechallenge, in addition to the criteria required for Grade 2(Figure 1). Of the 607,361 reports analyzed, 5,041 $(0.81 \%)$ met all of these requirements. An analysis of these reports' basic characteristics showed that $99.9 \%$ of welldocumented Grade 3 reports were 'investigation complete'. Detailed investigations were typically conducted by the regulatory authorities or pharmaceutical companies, suggesting that high report quality was obtained by supplementing information that had been missing. Analysis of report type showed that study reports accounted for 1,396 (27.7\%) of well-documented Grade 3 reports. Study reports are ADRs obtained from solicited studies such as drug-use results surveys and postmarketing clinical studies under the Japanese postmarketing safety measures. The quality of study reports may be affected by diverse factors, including whether a rechallenge was completed, the availability of detailed survey questionnaires, and visits by the company's medical representatives. The majority of the reporters and senders of welldocumented Grade 3 reports were physicians and pharmaceutical companies. This is because, in Japan, routinely, drug-use results surveys and/or other safety measures are requested by pharmaceutical companies from physicians.

\section{Comparison of the top ten adverse drug reactions and suspect drugs}

Comparing the top ten ADRs in well-documented Grade 3 reports with those in the overall reports revealed that disorders of hematopoietic function, such as leukopenia, neutropenia, and thrombocytopenia, occupied 4 of the top 10 places in both classifications. Interstitial lung disease and pneumonia were only observed in the overall reports. In contrast, fever, diarrhea, and decreased blood pressure were only observed in well-documented Grade 3 reports. In the overall reports, the top positions for suspect drugs were occupied by immunosuppressants. In contrast, among welldocumented Grade 3 reports, 8 of the top 10 suspect drugs were anticancer agents (the other 2 were nafamostat mesylate and irradiated platelet concentrate). The higher proportion of ADR reports related to disorders of hematopoietic function in well-documented and Grade 3 reports can be attributed to the higher proportion of reports with cytotoxic anticancer drugs in particular accounting for half of the top ten drugs. During treatment with cytotoxic anticancer drugs, leukopenia, neutropenia, or thrombocytopenia may occur due to bone marrow suppression.

If a drug holiday leads to recovery from bone marrow suppression by anticancer drugs, it is common practice to resume treatment at a reduced dose of the suspect drug. It is often difficult to replace anticancer drugs with other drugs because of differences in indications and evidence (even if allogeneic drugs with similar effects are available). They may be a factor favoring a rechallenge after the appearance of adverse events. In addition, new anticancer drugs are often the subject of specific use outcome studies. The collection of detailed safety information based on contracts between healthcare providers and companies may cause higher reporting quality. 
Table 2. Characteristics of analysis set

\begin{tabular}{|c|c|c|c|c|c|c|}
\hline \multirow{2}{*}{$\begin{array}{l}\text { VigiGrade } \\
\text { WHO documentation grading } \\
\text { scheme }\end{array}$} & \multicolumn{2}{|c|}{ Well-documented } & \multicolumn{4}{|c|}{ Not well-documented } \\
\hline & $\begin{array}{c}\text { Grade } 3 \\
(\mathrm{n}=5,041)\end{array}$ & $\begin{array}{c}\text { Grade } 2 \\
(n=315,616)\end{array}$ & $\begin{array}{l}\text { Grade } 3 \\
(n=137)\end{array}$ & $\begin{array}{c}\text { Grade } 2 \\
(n=13,086)\end{array}$ & $\begin{array}{c}\text { Grade } 1 \\
(n=39,912)\end{array}$ & $\begin{array}{c}\text { Grade } 0 \\
(n=233,569)\end{array}$ \\
\hline \multicolumn{7}{|l|}{ Reporters including } \\
\hline Physicians, n (\%) & $4,260(84.5)$ & $264,455(83.8)$ & $107(78.1)$ & $10,321(78.9)$ & $27,612(69.1)$ & $153,960(65.9)$ \\
\hline Pharmacists, $\mathrm{n}(\%)$ & $289(5.7)$ & $18,754(5.9)$ & $8(5.8)$ & $937(7.2)$ & $5,330(13.4)$ & $31,971(13.7)$ \\
\hline Physicians and Pharmacists, n (\%) & $252(5.0)$ & $17,541(5.6)$ & $6(4.4)$ & $634(4.8)$ & $2,345(5.9)$ & $8,340(3.6)$ \\
\hline Others, n (\%) & $240(4.8)$ & $14,866(4.7)$ & $16(11.7)$ & $1,194(9.1)$ & $4,625(11.6)$ & $39,298(16.8)$ \\
\hline \multicolumn{7}{|l|}{ Sender } \\
\hline Medical institution, $\mathrm{n}(\%)$ & $152(3.0)$ & $7,119(2.3)$ & $2(1.5)$ & $116(0.9)$ & $797(2.0)$ & $1,590(0.7)$ \\
\hline Pharmaceutical company, n (\%) & $4,889(97.0)$ & $308,497(97.7)$ & $135(98.5)$ & $12,970(99.1)$ & $39,115(98.0)$ & $231,979(99.3)$ \\
\hline \multicolumn{7}{|l|}{ Report status } \\
\hline Investigation complete, $\mathrm{n}(\%)$ & $5,040(99.9)$ & $315,213(99.9)$ & $137(100)$ & $13,031(99.6)$ & $39,780(99.7)$ & $232,531(99.6)$ \\
\hline Under investigation, $\mathrm{n}(\%)$ & $1(0.1)$ & $403(0.1)$ & $0(0)$ & $55(0.4)$ & $132(0.3)$ & $1,038(0.4)$ \\
\hline \multicolumn{7}{|l|}{ Report type } \\
\hline Spontaneous report, $\mathrm{n}(\%)$ & $3,468(68.8)$ & $220,143(69.7)$ & $123(89.8)$ & $11,803(90.2)$ & $33,054(82.8)$ & $202,718(86.7)$ \\
\hline Study report, n (\%) & $1,396(27.7)$ & $86,937(27.5)$ & $9(6.5)$ & $1,049(8.0)$ & $5,261(13.1)$ & $19,047(8.2)$ \\
\hline Other, n (\%) & $176(3.4)$ & $8,515(2.7)$ & $5(3.7)$ & $233(1.7)$ & $1,595(4.0)$ & $11,786(5.0)$ \\
\hline Unknown, n (\%) & $1(0.1)$ & $21(0.1)$ & $0(0)$ & $1(0.1)$ & $2(0.1)$ & $18(0.1)$ \\
\hline
\end{tabular}


Table 3. Missing elements in adverse drug reaction reports

\begin{tabular}{|c|c|c|c|c|c|c|}
\hline & \multicolumn{2}{|c|}{ Well-documented } & \multicolumn{4}{|c|}{ Not well-documented } \\
\hline & $\begin{array}{c}\text { Grade } 3 \\
(\mathrm{n}=5,041)\end{array}$ & $\begin{array}{c}\text { Grade } 2 \\
(n=315,616)\end{array}$ & $\begin{array}{l}\text { Grade } 3 \\
(n=137)\end{array}$ & $\begin{array}{c}\text { Grade } 2 \\
(n=13,086)\end{array}$ & $\begin{array}{c}\text { Grade } 1 \\
(\mathrm{n}=39,912)\end{array}$ & $\begin{array}{c}\text { Grade } 0 \\
(n=233,569)\end{array}$ \\
\hline Time-to-onset, $\mathrm{n}(\%)$ & $0(0)$ & $0(0)$ & $0(0)$ & $0(0)$ & $0(0)$ & $233,569(100)$ \\
\hline Onset date of ADR, n (\%) & $0(0)$ & $0(0)$ & $0(0)$ & $0(0)$ & $0(0)$ & $180,776(77.4)$ \\
\hline Onset date of treatment, $\mathrm{n}(\%)$ & $0(0)$ & $0(0)$ & $0(0)$ & $0(0)$ & $0(0)$ & $200,322(85.8)$ \\
\hline \multicolumn{7}{|l|}{ Time-to-onset penalty } \\
\hline $1.0, \mathrm{n}(\%)$ & $4,782(94.9 \%)$ & $293,508(93.0)$ & $33(24.1)$ & $3,839(29.3)$ & $29,623(74.2)$ & $0(0)$ \\
\hline $0.9, \mathrm{n}(\%)$ & $259(5.1 \%)$ & $22,108(7.0)$ & $9(6.6)$ & $1,292(9.9)$ & $6,304(15.8)$ & $0(0)$ \\
\hline $0.7, \mathrm{n}(\%)$ & $0(0)$ & $0(0)$ & $95(69.3)$ & $7,955(60.8)$ & $3,985(10.0)$ & $0(0)$ \\
\hline $0.5, \mathrm{n}(\%)$ & $0(0)$ & $0(0)$ & $0(0)$ & $0(0)$ & $0(0)$ & $233,569(100)$ \\
\hline Offset date of treatment, $\mathrm{n}(\%)$ & $426(8.5)$ & $38,089(12.1)$ & $36(26.3)$ & $4,461(34.1)$ & $11,351(28.4)$ & $198,005(84.8)$ \\
\hline Indication, $\mathrm{n}(\%)$ & $0(0)$ & $0(0)$ & $0(0)$ & $0(0)$ & $16,915(42.4)$ & $54,081(23.2)$ \\
\hline Outcome, n (\%) & $0(0)$ & $0(0)$ & $0(0)$ & $0(0)$ & $26,427(33.8)$ & $107,367(46.0)$ \\
\hline Sex, $\mathrm{n}(\%)$ & $0(0)$ & $0(0)$ & $10(7.3)$ & $1,510(11.5)$ & $970(2.4)$ & $25,104(10.8)$ \\
\hline Age, n (\%) & $0(0)$ & $0(0)$ & $32(23.4)$ & $4,268(32.6)$ & $2,495(6.3)$ & 34,765 (14.9) \\
\hline Dose, $\mathrm{n}(\%)$ & $408(8.1)$ & $29,835(9.5)$ & $38(27.7)$ & $3,846(29.4)$ & $10,412(26.1)$ & $130,538(55.9)$ \\
\hline Primary reporter, $\mathrm{n}(\%)$ & $208(4.1)$ & $10,397(3.3)$ & $8(5.8)$ & $543(4.2)$ & $1,452(3.6)$ & $9,835(4.2)$ \\
\hline Report type, n (\%) & $1(<0.1)$ & $21(<0.1)$ & $0(0)$ & $1(<0.1)$ & $2(<0.1)$ & $18(<0.1)$ \\
\hline
\end{tabular}

$A D R$, adverse drug reaction. 
Conversely, for interstitial lung disease, druginduced pneumonia, and lymphoproliferative disorders, it is generally not recommended to readminister the suspect drug once an adverse reaction has occurred from a safety standpoint. As a consequence, it is difficult to obtain information on rechallenges after these ADRs, and causality is often estimated by confirming symptom recovery with discontinuation of the suspect drug (16-17) (dechallenge positive).

The lowest quality, not well-documented Grade 0 reports tended to include immunosuppressive and anticoagulant drugs, as suspect drugs, and interstitial pneumonia, pneumonia, and lymphoproliferative disorders, as ADRs. Rheumatoid arthritis, methotrexate (MTX), and lymphoproliferative disorders (LPD) were the most common combination of the reason for the use of the suspect drug - suspect drug - and ADRs, with $89 \%$ of reports of this combination being not welldocumented Grade 0 (data not shown). MTXassociated lymphoproliferative disorders (MTXLPDs) occur during immunosuppressive drug treatment for diseases such as rheumatoid arthritis. MTX-LPDs may occasionally cause death. The 2011 edition of the Japan College of Rheumatology guideline for MTX use in patients with rheumatoid arthritis (17) described MTX-LPD. After the publication, the number of reports of MTX-LPD may have increased due to the increased public attention. Although over $90 \%$ of MTX-LPD related reports lacked the start date of suspect drug or onset date of ADR, the patient's sex, age, and outcome were recorded in $60-80 \%$ of these reports. MTX-LPD is a late-onset disease, and time-to-onset among Japanese rheumatoid arthritis was reported as approximately five years (18). Moreover, because MTX-LPDs include malignant diseases like cancer, it was challenging to identify the exact date of onset. Therefore, the lack of information concerning timeto-onset may be a consequence of these reasons. Nonetheless, time-to-onset information is indispensable for evaluating the causal relationship between the suspect drugs and ADRs, and other drug safety information.

\section{The proper use of two schemes}

Evaluation of the quality of reports in the JADER revealed that many reports lacked time-to-onset information as with other ADR databases $(19,20)$. Furthermore, the quality of reports varied depending on the reason for the suspect drug use - suspect drug
- ADR combination. When a causal relationship between suspect drugs and ADRs is assessed individually, it may be more efficient to extract and use reports classified as Grade 3 in the WHO documentation grading scheme because they include information on rechallenges. However, from our results, it was also demonstrated that the WHO documentation grading scheme, which required information on rechallenge for high-quality reporting, may result in the extraction of reports biased towards anticancer drugs and the ADRs associated with them. The vigiGrade completeness score would classify some ADR reports as low quality because of the lack of information on time-toonset, even if the reports contained beneficial patient characteristics. Moreover, the ADR reports without time-to-onset information included reports that had a long time-to-onset or secondary cancer for which the date of onset could not be determined. In summary, it was revealed that the two schemes need to be used appropriately, depending on the purpose of analysis, the target ADRs, and suspect drugs. For example, when a causal relationship between an anticancer drug and an ADR would be assessed, we can use well-documented reports in the vigiGrade due to their completeness. In all situations on postmarketing safety measures, information on time-to-onset is suggested to be essential to evaluate causality or to detect safety signals. In the JADER, a more advanced reporting system should be developed to gather this information precisely.

FUNDING. This research was partially supported by the Research on Regulatory Harmonization and Evaluation of Pharmaceuticals, Medical Devices, Regenerative and Cellular Therapy Products, Gene Therapy Products, and Cosmetics from the Japan Agency for Medical Research and Development (AMED).

CONFLICTS OF INTEREST. Masami Tsuchiya, Taku Obara, Makoto Miyazaki, Aoi Noda, Takamasa Sakai, Ryohkan Funakoshi, and Nariyasu Mano have no conflicts of interest directly relevant to the content of this study. Makoto Miyazaki is also an employee of a pharmaceutical company, MSDKK. However, he has no conflicts of interest directly relevant to the content of this study, as this study focuses on all marketed drugs in Japan and not on any specific drug.

\section{REFERENCES}

1. Inman WHW, Gill EP. Monitoring for Drug Safety, 2nd edition. Lancaster: MTP Press, 1986. 
2. Van Puijenbroek EP, van Grootheest K, Diemont WL, Leufkens HGM, Egberts ACG. Determinants of signal selection in a spontaneous reporting system for adverse drug reactions. Br J Clin Pharmacol. 2001;52(5):579586. https://dx.doi.org/10.1046\%2Fj.03065251.2001.01501.x

3. Pharmaceuticals and Medical Devices Agency, Japanese adverse drug event report database (JADER). https://www.pmda.go.jp/safety/infoservices/drugs/adr-info/suspected-adr/0005.html (in Japanese). Accessed 16 May 2020.

4. Lindquist $M$. VigiBase, the WHO Global ICSR Database System: Basic Facts. Drug Inf J. 2008;42(5), 409-419. https://doi.org/10.1177\%2F009286150804200501

5. US Food Drug Administration, FDA Adverse Event Reporting System (FAERS): latest quarterly data files. https:/www.fda.gov/drugs/questions-and-answersfdas-adverse-event-reporting-system-faers/fdaadverse-event-reporting-system-faers-latest-quarterlydata-files. Accessed 16 May 2020.

6. Netherlands pharmacovigilance centre Lareb. https://www.lareb.nl/en/. Accessed 16 May 2020.

7. European medicines agency, EudraVigirance: Access to EudraVigilance data. https://www.ema.europa.eu/en/humanregulatory/researchdevelopment/pharmacovigilance/eudravigilance/acces s-eudravigilance-data. Accessed 16 May 2020.

8. Anonymous. The WHO Adverse Reactions Database On-line Searches User's Manual. Uppsala Monitoring Centre, 1997.

9. Bergvall T, Norén GN, Lindquist M. vigiGrade: a tool to identify well-documented individual case reports and highlight systematic data quality issues. Drug Saf. 2014;37(1):65-77. https://doi.org/10.1007/s40264013-0131-x

10. Bres V, Robin P, Pinzani V, Philibert C, Bos-Thompson MA, Hillaire-Buys D, Faillie JL. The ATHE score: a new indicator for data quality management of spontaneous reporting in PharmacoVigilance. Drug Saf. 2013;36(9)916 (abstr. ISP3780-46).

11. Alshammari TM, Al-Kathiri WH, Le Louet $H$, Aljadhey HS. Completeness of adverse drug reactions reports of the Saudi adverse event reporting system. Saudi Med J. 2015;36(7):821-828. https://doi.org/10.15537/smj.2015.7.11751.

12. Sánchez-Sánchez B, Altagracia-Martínez M, KravzovJinich J, Moreno-Bonett C, Vázquez-Moreno E,
Martínez-Núñez JM. Evaluation of completeness of suspected adverse drug reaction reports submitted to the Mexican national pharmacovigilance centre: a cross-sectional period-prevalence study. Drug Saf. 2012;35(10):837-844.

https://doi.org/10.1007/bf03261979

13. Mahajan MM, Thatte UM, Gogtay NJ, Deshpande S. An analysis of completeness and quality of adverse drug reaction reports at an adverse drug reaction monitoring centre in Western India. Perspect Clin Res. 2018;9(3):123-126. https://doi.org/10.4103/picr.picr_105_17

14. Tsuchiya M, Obara T, Sakai T, Nomura K, Takamura C, Mano N. Quality evaluation of the Japanese Adverse Drug Event Report database (JADER). Pharmacoepidemiol Drug Saf. 2020;29(2):173-181. https://doi.org/10.1002/pds.4944

15. Tsuchiya M, Obara T, Miyazaki M, Noda A, Takamura C, Mano N. The quality assessment of the Japanese Adverse Drug Event Report database using vigiGrade. Int J Clin Pharm. 2020;42(2):728-736. https://doi.org/10.1007/s11096-020-00969-7

16. Ministry of Health, Labour and Welfare, Response Manual by Serious Adverse Disease Interstitial pneumonia (pneumonitis, septicaemia, and pulmonary fibrosis), $\quad$ November 2006. http://www.info.pmda.go.jp/juutoku/file/jfm0611002. pdf (in Japanese). Accessed 16 Mar 2020.

17. Japan College of Rheumatology guideline for the use of methotrexate in patients with rheumatoid arthritis, 2016 revised edition, https://www.ryumachijp.com/publication/pdf/MTX2016kanni.pdf (in Japanese). Accessed 16 Mar 2020.

18. Hoshida Y, Xu JX, Fujita S, Nakamichi I, Ikeda J, Tomita Y, Nakatsuka S, Tamaru J, Iizuka A, Takeuchi T, Aozasa K. Lymphoproliferative disorders in rheumatoid arthritis: clinicopathological analysis of 76 cases in relation to methotrexate medication. J Rheumatol. 2007;34(2):322-331.

19. McCarthy M. Drug makers' adverse event reports are often incomplete, US report finds. Bmj Br Medical J. 2015;350(feb04 6):h651.

20. Plessis L, Gómez A, García N, Cereza G, Figueras A. Lack of essential information in spontaneous reports of adverse drug reactions in Catalonia - a restraint to the potentiality for signal detection. Eur J Clin Pharmacol. 2017;73(6):751-758. 


\section{SUPPLEMENTS}

2019

2020

\begin{tabular}{ll|} 
Drug start date & Feb 2019 \\
ADR onset date & $\begin{array}{r}\text { TTO: } 2 \text { to } 4 \text { months } \\
\text { No penalty }\end{array}$ \\
\hline
\end{tabular}

$\begin{array}{lll}\text { Drug start date } & \text { Feb 2019 } \\ \text { ADR onset date } & \text { Feb 2019 } & \begin{array}{r}\text { TTO: }-1 \text { to }+1 \text { month } \\ \text { Penalty } 10 \%\end{array} \\ \end{array}$

Drug start date

2019

ADR onset date
TTO: -2 to +11 months Penalty $30 \%$

Drug start date

ADR onset date

Feb 2019

TTO: ?

Penalty $50 \%$

Figure S1. Four examples of penalties when there is imprecise or missing information on time-to-onset. $A D R$ adverse drug reaction, TTO time-to-onset 
Table S1. Dimensions (weighted by their relative importance for causality assessment and follow the listed principles) and penalties (for absence of information on specific dimensions reflecting the importance of that dimension for causality assessment) based on vigiGrade and the present study

\begin{tabular}{|c|c|c|c|c|}
\hline Dimension & Description & Considerations & Penalty based on vigiGrade & Penalty for the present study \\
\hline Time-to-onset & $\begin{array}{l}\text { Time from treatment start to the } \\
\text { suspected ADR }\end{array}$ & $\begin{array}{l}\text { Imprecise information penalised if there is } \\
\text { ambiguity as to whether the drug preceded } \\
\text { the adverse event }\end{array}$ & $\begin{array}{l}50 \% ; 30 \% \text { if the uncertainty } \\
\text { Exceeds } 1 \text { month } \\
10 \% \text { otherwise (See Figure S2) }\end{array}$ & $\begin{array}{l}50 \% ; 30 \% \text { if the uncertainty exceeds } 1 \\
10 \% \text { otherwise }\end{array}$ \\
\hline Indication & $\begin{array}{l}\text { Indication for treatment with the } \\
\text { drug }\end{array}$ & $\begin{array}{l}\text { Penalty imposed if information is missing or } \\
\text { cannot be mapped to standard terminologies } \\
\text { such as ICD or MedDRA }\end{array}$ & $30 \%$ & $30 \%$ \\
\hline Outcome & $\begin{array}{l}\text { Outcome of the adverse event in } \\
\text { this patient }\end{array}$ & & $30 \%$ & $30 \%$ \\
\hline Sex & Patient sex & 'Unknown' treated as missing & $30 \%$ & $30 \%$ \\
\hline Age & $\begin{array}{l}\text { Patient's age at onset of the } \\
\text { suspected ADR }\end{array}$ & Age 'unknown' treated as missing & $\begin{array}{l}30 \% ; 10 \% \text { if only the age group was } \\
\text { specified }\end{array}$ & $\begin{array}{l}30 \% ; 10 \% \text { if only the generational } \\
\text { group was specified (e.g., elderly, } \\
\text { neonate, child) }\end{array}$ \\
\hline Dose & Dose of the drug(s) & & $10 \%$ & $10 \%$ \\
\hline Country & Country of origin & $\begin{array}{l}\text { Supportive in causality assessment since medical } \\
\text { practice and adverse reaction reporting vary } \\
\text { between countries }\end{array}$ & $10 \%$ & Not applicable \\
\hline \multirow[t]{2}{*}{ Primary reporter } & $\begin{array}{l}\text { Occupation of the person who } \\
\text { reported the case } \\
\text { (e.g., physician, pharmacist) }\end{array}$ & $\begin{array}{l}\text { Supportive in causality assessment since the } \\
\text { interpretation of reported information may differ } \\
\text { depending on the reporter's qualifications }\end{array}$ & $10 \%$ & $10 \%$ \\
\hline & & $\begin{array}{l}\text { 'Unknown' penalised as missing information, but } \\
\text { 'other' not penalized. }\end{array}$ & & \\
\hline Report type & $\begin{array}{l}\text { Type of report (e.g., spontaneous } \\
\text { report, report from study, other) }\end{array}$ & & $10 \%$ & $10 \%$ \\
\hline Comments & Free-text information & Uninformative text snippets excluded & $10 \%$ & Not applicable \\
\hline
\end{tabular}

ADR, adverse drug reaction; ICD, international statistical classification of disease and related health problems; MedDRA, medical dictionary for regulatory activities. 
Table S2. The top ten suspect drugs in the overall reports and in well-documented Grade 3 reports

\begin{tabular}{llll}
\hline Overall reports $(\mathrm{n}=607,361)$ & & & Well-documented Grade 3 ( $\mathrm{n}=5,041)$ \\
\cline { 1 - 1 } Name of suspect drugs & $\mathrm{N}(\%)$ & Name of suspect drugs \\
\hline Methotrexate & $13,539(2.2)$ & Cisplatin & $\mathrm{N}(\%)$ \\
Tacrolimus hydrate & $8,585(1.4)$ & Paclitaxel & 174 (3.5) \\
Bevacizumab (genetical recombination) & $8,086(1.3)$ & Sorafenib tosylate & Amrubicin hydrochloride \\
Prednisolone & $7,076(1.2)$ & Bevacizumab (genetical recombination) \\
Nivolumab (generical recombination) & $6,572(1.1)$ & Tegafur, Gimeracil, Oteracil potassium \\
Apixaban & $6,010(1.0)$ & Nafamostat mesylate \\
Rivaroxaban & $5,724(0.9)$ & 135 (2.7) \\
Cyclosporine & $5,451(0.9)$ & 106 (2.1) & 105 (2.1) \\
Oxaliplatin & $5,378(0.9)$ & Irradiated Platelet Concentrate \\
Tegafur, Gimeracil, Oteracil potassium & $5,105(0.8)$ & Dasatinib hydrate \\
\hline
\end{tabular}


J Pharm Pharm Sci (www.cspsCanada.org) 24, 161 - 173, 2021

Table S3. The top ten ADRs in the overall reports and in well-documented Grade 3 reports

\begin{tabular}{|c|c|c|c|}
\hline Overall reports $(\mathrm{n}=607,361)$ & & Well-documentec & \\
\hline Adverse reaction & $n(\%)$ & Adverse reaction & $n(\%)$ \\
\hline Interstitial lung disease & $25,203(4.2)$ & White blood cell count decreased & $333(6.6)$ \\
\hline Anaphylactic shock & $13,183(2.2)$ & Neutrophil count decreased & $261(5.2)$ \\
\hline Hepatic function abnormal & $12,776(2.1)$ & Pyrexia & $168(3.3)$ \\
\hline Platelet count decreased & $9,240(1.5)$ & Anaphylactic shock & $160(3.2)$ \\
\hline Pneumonia & $8,729(1.4)$ & Hepatic function abnormal & $156(3.1)$ \\
\hline Liver disorder & $8,033(1.3)$ & Platelet count decreased & $152(3.0)$ \\
\hline Neutrophil count decreased & $7,358(1.2)$ & Neutropenia & $127(2.5)$ \\
\hline Drug eruption & $6,782(1.1)$ & Diarrhoea & $113(2.2)$ \\
\hline White blood cell count decreased & $6,527(1.1)$ & Blood pressure decreased & $113(2.2)$ \\
\hline Neutropenia & $6,353(1.1)$ & Drug eruption & $102(2.0)$ \\
\hline
\end{tabular}


Table S4. The top ten adverse drug reaction - suspect drug combinations in well-documented grade 3 and in not well-documented Grade 0 reports

\begin{tabular}{|c|c|c|c|}
\hline \multirow{2}{*}{$\begin{array}{l}\text { Well-documented Grade } 3(n=5,041) \\
\text { Adverse reaction + suspect drug }\end{array}$} & \multirow[b]{2}{*}{$n(\%)$} & \multicolumn{2}{|l|}{ Not well-documented Grade $0(n=233,569)$} \\
\hline & & Adverse reaction + suspect drug & $\mathrm{n}(\%)$ \\
\hline White blood cell count decreased + Paclitaxel & $61(1.2)$ & Lymphoproliferative disorder + Methotrexate & $2,988(1.3)$ \\
\hline Blood pressure decreased + Irradiated Red Blood Cells & $48(1.0)$ & Heparin-induced thrombocytopenia + Heparin Sodium & $675(0.3)$ \\
\hline White blood cell count decreased + Amrubicin hydrochloride & $43(0.9)$ & Urinary retention + Fesoterodine Fumarate & $596(0.3)$ \\
\hline Neutrophil count decreased + Amrubicin hydrochloride & $40(0.8)$ & Osteonecrosis of jaw + Zoledronic Acid Hydrate & $584(0.3)$ \\
\hline Neutrophil count decreased + Bevacizumab (genetical recombination) & $37(0.7)$ & Hypoglycemia + Glimepiride & $580(0.3)$ \\
\hline White blood cell count decreased + Nogitecan hydrochloride & $36(0.7)$ & Interstitial lung disease + Methotrexate & $557(0.2)$ \\
\hline Anaphylactic shock + Nafamostat mesylate & $35(0.7)$ & $\begin{array}{l}\text { Drug reaction with eosinophilia and systemic symptoms + } \\
\text { Carbamazepine }\end{array}$ & $529(0.2)$ \\
\hline Shock + Nafamostat mesylate & $27(0.5)$ & Diffuse large B-cell lymphoma + Methotrexate & $483(0.2)$ \\
\hline Urticaria + Irradiated Platelet Concentrate & $27(0.5)$ & Lymphoma + Methotrexate & $457(0.2)$ \\
\hline & $26(0.5)$ & Pancytopenia + Methotrexate & $456(0.2)$ \\
\hline Hepatic function abnormal + Sorafenib tosylate & & & \\
\hline
\end{tabular}

